\title{
Tratamento da Incontinência Urinária em Mulheres com Esclerose Múltipla (EM): Série de Casos
}

\author{
Treatment of Urinary Incontinence in Woman with Multiple Sclerosis (MS): Case Series \\ Cintia Elisabete Fischer Blosfeld ${ }^{1}$, Sandra Dias de Souza ${ }^{2}$
}

\begin{abstract}
RESUMO
Introduçáo. Cerca de 50 a 90\% dos pacientes com Esclerose Múltipla (EM) apresentam sintomas urinários, dentre eles incontinência, frequência ou urgência urinária. Destaca-se a importância em estudar modalidades terapêuticas que possam corrigir ou ao menos atenuar tais sintomas. Objetivo. Analisar os benefícios do Biofeedback de pressão no tratamento da incontinência urinária de mulheres com diagnóstico clínico de EM. Método. Trata-se de um estudo experimental do tipo série de casos realizado com 8 mulheres submetidas a 10 atendimentos com o Biofeedback de pressão. Para análise da evoluçáo das mulheres foram realizadas avaliaçôes, antes e depois do tratamento, em relação à força muscular do assoalho pélvico e qualidade de vida através da aplicação do questionário King's Health Questionnaire. Resultados. Foram encontrados valores significantes na Avaliação Funcional do Assoalho Pélvico $(p=0,018)$, na Contração Rápida Máxima $(p=0,012)$, na Contração Prolongada $(p=0,05)$ e na Resistência $(\mathrm{p}=0,05)$. Na avaliação da qualidade de vida somente os domínios "percepção geral de saúde" e "limitaçóes sociais" não foram significantes. Conclusáo. Verificou-se que o biofeedback de pressão promoveu bons resultados no tratamento de pacientes com EM. Sua aplicação permitiu uma maior conscientização e força da musculatura do assoalho pélvico e melhora da qualidade de vida das pacientes.
\end{abstract}

Unitermos. Incontinência Urinária, Esclerose Múltipla, Biofeedback.

Citaçáo. Blosfeld CEF, Souza SD. Tratamento da Incontinência Urinária em Mulheres com Esclerose Múltipla (EM): Série de Casos.

\footnotetext{
Trabalho realizado nas Clínicas Integradas da Faculdade Evangélica do Paraná, Curitiba-PR, Brasil.

1.Graduada em Fisioterapia pela Faculdade Evangélica do Paraná, Pós Graduada em Fisioterapia Neurofuncional pela Universidade Tuiuti do Paraná, Curitiba-PR, Brasil.

2.Graduada em Fisioterapia pela Universidade Tuiuti do Paraná, Mestre em Ergonomia, UFSC, Docente da Universidade Tuiuti do Paraná, Docente Especialização Lato Sensu em Fisioterapia Neurofuncional, Curitiba-PR, Brasil.
}

\begin{abstract}
Introduction. Around 50-90\% of patients with Multiple Sclerosis (MS) have urinary symptoms: incontinence, frequency or urinary urgency. Emphasize the extreme importance in studying therapeutic modalities that can correct or at least alleviate such symptoms. Objective. Investigate the benefits of pressure biofeedback treatment of urinary incontinence woman diagnosed with MS. Method. This is an experimental study of the case series conducted with eight woman that were subjected to 10 attendance with Biofeedback pressure. To analyze the evolution, the women were evaluated, before and after the treatment, of the relation to the pelvic floor muscle strength and quality of life by applying the questionnaire King's Health Questionnaire. Results. We found statistically significant gains in Functional Evaluation of the Pelvic Floor ( $\mathrm{p}=0.018)$ in the Quick Maximum Contraction $(\mathrm{p}=0.012)$, the Prolonged Contraction $(\mathrm{p}=0.05)$ and Resistance $(\mathrm{p}=0.05)$. In evaluating the quality of life only the domains "General Health Perception" and "Social Constraints" did not possess significant differences. Conclusion. It was found that Biofeedback pressure promoted good results in treating patients with MS. Its application has enabled to greater awareness and strength of pelvic floor muscle and improved quality of life of patients.
\end{abstract}

Keywords. Urinary Incontinence, Multiple Sclerosis, Biofeedback.

Citation. Blosfeld CEF, Souza SD. Treatment of Urinary Incontinence in Woman with Multiple Sclerosis (MS): Case Series.
Endereço para correspondência: Cíntia Elisabete Fischer Blosfeld Rua dos Funcionários, 144 Bl. 3 apt. 402. Bairro Cabral CEP 80035-050, Curitiba-PR, Brasil. E-mail: cintiaef@gmail.com 


\section{INTRODUÇÃO}

A Esclerose Múltipla (EM) é uma doença desmielinizante crônica caracterizada por múltiplas lesôes na substância branca do Sistema Nervoso Central (SNC). Tais lesôes incluem áreas de inflamação, desmielinização e formação de cicatrizes gliais ${ }^{1}$.

As manifestaçóes clínicas da EM dependem da localização das áreas de esclerose, as quais podem ocorrer, por exemplo, no quiasma óptico, no tronco, no cerebelo e no cérebro. A partir da localização das áreas de esclerose podem surgir vários sinais e sintomas, desde alteraçóes visuais, motoras, sensitivas, psiquiátricas e cognitivas, até sintomas urinários, os quais são o foco principal deste trabalho ${ }^{1}$.

Dentre os sintomas urinários destaca-se incontinência, frequência e/ou urgência urinária ${ }^{1}$, presentes em cerca de $50 \%$ a $90 \%$ dos pacientes com $\mathrm{EM}^{2}$. Tais disfunçôes são características de bexiga neurogênica, decorrentes da disfunção uretral ou do detrusor, causadas por lesōes neurológicas, como no caso da EM. Neste caso, pode haver lesões de áreas responsáveis pela inibição do detrusor, levando a contraçóes involuntárias do mesmo, ocasionando a incontinência urinária (IU) ${ }^{3}$.

Em virtude da implicação de tais disfunçôes à vida dos pacientes portadores de EM, é de extrema relevância estudar métodos que possam corrigir ou ao menos atenuar os sintomas urinários no paciente com EM, visto que a IU é considerada, segundo a International Continence Society, toda e qualquer perda involuntária de urina, a qual por consequência constitui um problema social ou de higiene ${ }^{4}$.

Deste modo, propóe-se com este estudo, analisar a eficácia do Biofeedback de pressão no tratamento da IU do paciente com EM.

O Biofeedback é um método de reeducação que constitui a aplicação do feedback ao homem ${ }^{5,6}$. Seu objetivo é modificar uma resposta fisiológica inadequada ou propiciar a aquisição de uma nova resposta fisiológica ${ }^{6}$. Segundo o I Consenso Brasileiro de Incontinência Urinária, Uroneurologia e Disfunçóes Miccionais, é o monitoramento, através de aparelhos, de eventos fisiológicos que o paciente é incapaz de distinguir por si só ${ }^{7}$. Para isso, exige a participação contínua do paciente em sua reeducação pelo fato de ser uma técnica exclusivamente ativa, onde o aparelho apenas registra e amplifica a atividade muscular exercida pelo paciente sob a forma de sinais sensoriais (visual e auditivo $)^{5,6}$.

Para tratamento das disfunçóes miccionais utilizase dois tipos de Biofeedback: o eletromiográfico e o de pressão. O Biofeedback eletromiográfico possui um sensor na derme que detecta a atividade elétrica que é liberada pelos músculos do assoalho pélvico quando ocorre a contração e processa esse sinal de forma sonora ou visual para o paciente ${ }^{8}$.

O Biofeedback de pressão também proporciona ao paciente a reeducação da Musculatura do Assoalho Pélvico (MAP) através de sinais luminosos, numéricos e sonoros. Esses sinais permitem a auto-avaliação da MAP sobre o movimento a ser medido, ensinado e aprendido e proporciona maior consciência da contração muscular ${ }^{8}$.

O feedback é dado ao paciente quando este contrai ou relaxa a MAP. É colocada uma sonda inflável no canal vaginal (mulheres) ou anal (homens) e esta detecta variaçóes de pressão em centímetros de água $\left(\mathrm{cmH}_{2} \mathrm{O}\right)$, mostradas ao mesmo no visor do aparelho ${ }^{8}$. A partir dessas informaçóes que o paciente poderá ter maior consciência da MAP, podendo reaprender a contraí-la ativamente e manter a continência urinária. A presença de um profissional é muito importante durante a aplicação do biofeedback, para orientar a correta realizaçáo da contração muscular e evitar distraçóes que possam dificultar o aprendizado 5 .

São amplamente discutidos na literatura os resultados significativos do biofeedback em pacientes sem desordens neurológicas ${ }^{9}$. Porém, pouco se encontra sobre tal modalidade terapêutica no paciente com EM.

Tem-se relatado também na literatura formas conservadoras de tratamento, dentre elas, cateterismo intermitente, drogas anticolinérgicas e toxina botulínica ${ }^{10}$. Porém, há um número reduzido de publicações referente à temática da presente pesquisa, fato este que faz surgir o seguinte problema: - Será que o Biofeedback de Pressão é eficiente para auxiliar no tratamento da incontinência urinária do paciente portador de EM?

\section{MÉTODO}

\section{Amostra}

Trata-se de um estudo experimental do tipo série de casos desenvolvido no Laboratório de Uroginecologia 
das Clínicas Integradas da Faculdade Evangélica do Paraná (FEPAR) no período entre janeiro e junho de 2010.

Foram recrutados para o estudo indivíduos com idade entre 20 e 55 anos, sem distinção de gênero, com diagnóstico clínico de Esclerose Múltipla, independentemente do tipo (Surto-remissão, Primária progressiva ou Secundária progressiva). Porém a população masculina não foi alcançada, levando ao término do estudo apenas com mulheres. Tais voluntárias deveriam ser previamente avaliadas clinicamente por um Neurologista com experiência na área de doenças desmielinizantes e apresentar placas de desmielinização em estudo de imagem (Ressonância Magnética), além de apresentar sintomas urinários característicos de bexiga neurogênica hiperativa (urgência, frequência e/ou urgeincontinência urinária).

Foram excluídas do estudo voluntárias com menos de 20 anos, ou mais de 55 anos que apresentaram sintomas urinários do tipo bexiga neurogênica flácida ou que não apresentaram placas de desmielinização em estudo de imagem (Ressonância Magnética); mulheres com prolapso grau III, cirurgia pélvica em um período menor do que seis meses; portadoras de marcapasso ou dispositivo contraceptivo (DIU); gestantes; ou voluntárias com tumor intrapélvico e/ou infecção urinária.

Após a descrição dos critérios de inclusão e exclusão foram atendidas 8 voluntárias com média de idade de $46 \pm 8,16$ anos. Além das 8 voluntárias atendidas, 5 mulheres foram excluídas do estudo por não se encaixarem nos critérios de inclusão ou por apresentarem surtos da doença durante o tratamento. Os homens não aderiram à pesquisa apesar da possibilidade de realização da intervenção.

Cada voluntária, antes de realizar os procedimentos de avaliação e tratamento, foi informada quanto aos objetivos da pesquisa e assinou o Termo de Consentimento Livre e Esclarecido (TCLE), de acordo com as normas e diretrizes da resolução CSN 196/96 e suas complementares. O projeto foi aprovado pelo Comitê de Ética em Pesquisa da Universidade Tuiuti Paraná (Protocolo $n^{\circ}$ 000130/2009).

\section{Procedimento}

As voluntárias foram submetidas aos procedimentos de avaliação inicial e final, que incluiu: anamnese (identificação, tempo de diagnóstico da EM, tipo de
EM, tratamentos realizados, queixas e sintomas urinários, questionamentos quanto a momentos de perda de urina, patologias associadas, hábitos de vida, antecedentes ginecológicos e obstétricos); exame físico (aspecto da pele e mucosas, reflexos, Avaliação Funcional do Assoalho Pélvico - AFA e Perineometria); e avaliação da qualidade de vida através do King's Health Questionnaire.

Antes de qualquer intervenção todas as voluntárias receberam informaçóes em relação à forma de avaliação, tratamento e possíveis desconfortos para que não houvesse quaisquer constrangimentos. Foram orientadas também quanto à musculatura do assoalho pélvico e suas funções relacionadas ao controle miccional.

A AFA avalia a força de contração da musculatura do assoalho pélvico (MAP). Para sua realização as voluntárias permaneceram em posição ginecológica e o examinador introduziu os dedos indicador e médio no eixo vaginal ${ }^{6}$. Foi então solicitada uma contração da MAP e a notação se deu em graus de 0 a 5 (Escala de Oxford), onde 0 não há função perineal objetiva, nem mesmo à palpação; e 5 a função perineal é objetiva e possui resistência opositora mantida à palpação ${ }^{11}$.

A Perineometria foi realizada através do Perina Biofeedback da marca Quark. Sua função é medir a força e sustentação da MAP ${ }^{12}$. Para sua realização as voluntárias permaneceram em posição ginecológica, então foi introduzida uma sonda vaginal inflável recoberta por um preservativo sem lubrificante com um gel lubrificante a base de água da marca $K Y^{\circledast}$. Após inserção da sonda, a válvula do insuflador foi fechada e a sonda inflada suavemente, até que a voluntária sentisse o contato contra a parede vaginal. Após tal sequência o aparelho foi ligado e ajustado na posição zero.

A avaliação da MAP através do perineômetro foi realizada em três etapas:

1. Avaliação da Contração Rápida Máxima - avalia a capacidade de fechamento do esfíncter: solicitou-se à voluntária 3 contraçôes rápidas e máximas. Para análise dos dados foi calculada a média entre elas ${ }^{13}$;

2. Avaliação da Contração Prolongada - demonstra a capacidade de manter as vísceras do assoalho pélvico: solicitou-se à voluntária contraçóes mantidas o máximo de tempo possível. Foi registrado o tempo e a pressão da contração mantida. O teste era interrompido quando 
a voluntária não conseguisse manter as contraçóes iguais à inicial, em 50\% do tempo ou do valor da pressão registrados inicialmente. Para análise dos dados foi calculado o tempo médio de contração;

3. Avaliação do maior número de contrações realizadas - avalia a resistência das fibras de contração rápida: solicitou-se à voluntária contraçôes rápidas mantidas durante 1 segundo. $\mathrm{O}$ teste era interrompido quando a voluntária não mais atingisse no mínimo $50 \%$ da pressão de contração inicial. Para análise dos dados foi registrado o número de repetiçóes.

As etapas 2 e 3 foram criadas para avaliar outros tipos de contração além da contração rápida máxima citada por Chiarapa. Afinal, para que se possa criar um programa de tratamento individualizado para cada paciente também é necessário saber o tempo de contração prolongada e a resistência da MAP de cada paciente.

Ao final da avaliação da Perineometria, a sonda foi desinsuflada e, em seguida, retirada da voluntária para então ser realizada assepsia do material e da voluntária.

A avaliação da qualidade de vida foi realizada através do King's Health Questionnaire, questionário que analisa a presença de sintomas de IU e seu impacto nos vários aspectos da individualidade na qualidade de vida. O questionário contém 30 questóes subdivididas em nove domínios. Referem-se, respectivamente, a percepção da saúde, o impacto da incontinência, as limitaçóes do desempenho das tarefas, a limitação física, a limitação social, o relacionamento pessoal, as emoçóes, o sono e a energia e as medidas de gravidade. Existe também uma escala de sintomas que é composta pelos seguintes itens: frequência urinária, noctúria, urgência, hiperreflexia vesical, incontinência urinária de esforço, enurese noturna, incontinência no intercurso sexual, infecçôes urinárias e dor na bexiga ${ }^{14}$.

Após a avaliação inicial, as voluntárias foram submetidas a 10 atendimentos, com a utilização do Biofeedback de pressão.

O Biofeedback de pressão é uma forma de tratamento também realizada através do aparelho PerinaBiofeedback da marca Quark, o qual possui duas funçóes distintas: Avaliação (Perineometria) e Tratamento (Biofeedback de pressão).

Para realização do tratamento com o Biofeedback de pressão, os procedimentos se assemelham aos da avaliação, porém com objetivos distintos. Para realização do procedimento, as voluntárias permaneceram em posição ginecológica. Entáo era introduzida uma sonda vaginal inflável recoberta por um preservativo sem lubrificante com um gel lubrificante a base de água da marca $\mathrm{KY}^{\odot}$. Após inserção da sonda, a válvula do insuflador era fechada e a sonda inflada suavemente, até que a voluntária sentisse o contato contra a parede vaginal.

Os parâmetros aplicados são os do aparelho Perina-Biofeedback, incluindo: Objetivo, determinada pressão em $\mathrm{cmH}_{2} \mathrm{O}$ que deve ser alcançada para que se inicie o tempo de contração; Work, ou tempo de manutenção da contração, que foi de 4 segundos; e Rest, ou tempo de relaxamento entre cada contração, que foi de 4 segundos. O objetivo a ser cumprido a cada dia foi de $50 \%$ da primeira contração máxima do mesmo dia. As voluntárias fizeram o treinamento durante 30 minutos, na frequência de 3 vezes semanais no período da tarde. Tais parâmetros foram pré-estabelecidos pela Pesquisadora responsável e Equipe de Pesquisadores respeitando o fato de que as pacientes com EM apresentam fatigabilidade importante e não teriam condições de realizar mais do que 30 minutos de terapia diária, nem mesmo realizar o tratamento diariamente. Também foi respeitado o período do dia (tarde) para realização da terapia para que não houvesse interferência em relação à fatigabilidade em outros períodos.

Após a aplicação do Biofeedback de pressão, as voluntárias foram reavaliadas e novamente responderam o questionário de qualidade de vida King's Health Questionnaire.

\section{Análise Estatística}

Foi utilizado o teste de Wilcoxon para dados pareados para verificar se existiam diferenças entre os momentos pré e pós intervenção na Avaliação Funcional do Assoalho Pélvico, nos três tipos de contração avaliados na Perineometria e nos nove domínios do King's Health Questionnaire, comparando antes e depois da intervenção.

A escala de sintomas inserida no King's Health Questionnaire foi analisada de forma descritiva através de uma tabela de frequências.

As análises estatísticas e confecção dos gráficos foram efetuados com o pacote estatístico Statistica. 
RESULTADOS

Foram atendidas 8 voluntárias com média de idade de 46 $\pm 8,16$ anos. Na Tabela 1 são listadas suas características demográficas e antropométricas.

Tabela 1

Características demográficas e antropométricas das voluntárias

\begin{tabular}{l|l|c}
\hline Variável & $\begin{array}{c}\text { Média } \pm \text { Desvio } \\
\text { Padráo/Frequência }\end{array}$ \\
\hline Idade (anos) & $46,00 \pm 8,16$ \\
Peso (kg) & $71,88 \pm 23,44$ \\
Altura (m) & $1,54 \pm 0,04$ \\
IMC & $30,50 \pm 10,45$ \\
Tempo de Diagnóstico (Anos) & $9,25 \pm 3,85$ \\
\hline Tipo Esclerose & Surto Remissão & $8(100 \%)$ \\
Múltipla & Primária Progressiva & 0 \\
\multicolumn{2}{l|}{} & 0 \\
\hline Sexo & Secundária & 0 \\
& Progressiva & $8(100 \%)$ \\
\hline Etnia & Masculino & $3(37,5 \%)$ \\
& Feminino & $1(12,5 \%)$ \\
& Branca & $4(50 \%)$ \\
\hline
\end{tabular}

Houve diferenças entre os momentos pré e pós intervenção para a Avaliação Funcional do Assoalho Pélvico e para as três formas de observaçáo da Perineometria (Tabela 2).

$\mathrm{Na}$ Avaliaçáo Funcional do Assoalho Pélvico as voluntárias apresentaram $2,75 \pm 1,39$ antes do tratamento, e $4,13 \pm 0,64$ depois do tratamento $(\mathrm{p}=0,018)$.

Na Perineometria - Contração Rápida Máxima $\left(\mathrm{cmH}_{2} \mathrm{O}\right)$ as voluntárias apresentaram 5,29 $\pm 3,32$ antes do tratamento, e $16,79 \pm 12,95$ depois do tratamento $(\mathrm{p}=0,012)$.
$\mathrm{Na}$ Perineometria - Contração Prolongada (segundos) as voluntárias apresentaram 9,03 $\pm 6,08$ antes do tratamento, e 20,66 $\pm 14,45$ depois do tratamento $(\mathrm{p}=0,05)$.

$\mathrm{Na}$ Perineometria - Resistência (número de repetiçóes) as voluntárias apresentaram $14,13 \pm 14,83$ antes do tratamento, e $31,75 \pm 12,93$ depois do tratamento $(\mathrm{p}=0,05)$.

Após a avaliação dos 9 domínios do King's Health Questionnaire verificou-se que somente os domínios "percepção geral de saúde" e "limitações sociais" não possuíram diferenças significantes entre os momentos pré e pós intervenção (Tabela 3).

Para análise da escala de sintomas que consta no King's Health Questionnaire foi realizada uma análise das frequências absolutas e percentuais, evidenciados na Tabela 4 .

\section{DISCUSSÃO}

Os resultados do presente estudo demonstraram que os pacientes com EM podem apresentar atenuação dos sintomas urinários característicos da bexiga neurogênica do tipo hiperativa através da utilização do Biofeedback de pressão. Isto sugere que também nos pacientes com desordens neurológicas a contração ativa da MAP provoca um relaxamento do detrusor através do reflexo inibitório períneo detrusor descrito por Mahony ${ }^{15}$.

Tal situação já é evidenciada em pacientes sem desordens neurológicas, porém com bexiga hiperativa. Um estudo realizado com 28 voluntários demonstrou através de exames cistométricos que a contração ativa da musculatura do assoalho pélvico provoca um aumento da pressão uretral e uma diminuição da pressão vesical ${ }^{16}$. A partir disso, acredita-se que a urgência miccional diminui por mecanismo reflexo inibitório sobre o detrusor,

Tabela 2

Valores de média \pm desvio padrão da Avaliação Funcional do Assoalho Pélvico e da Perineometria nos momentos pré e pós intervenção

\begin{tabular}{lccc}
\hline Variável & Pré & Pós & p \\
\hline Avaliação Funcional do Assoalho Pélvico & $2,75 \pm 1,39$ & $4,13 \pm 0,64$ & $0,018^{*}$ \\
Perineometria - Contração Rápida Máxima $\left(\mathrm{cmH}_{2} \mathrm{O}\right)$ & $5,29 \pm 3,32$ & $16,79 \pm 12,95$ & $0,012^{*}$ \\
Perineometria - Contração Prolongada (segundos) & $9,03 \pm 6,08$ & $20,66 \pm 14,45$ & $0,050^{*}$ \\
Perineometria - Resistência (número de repetiçōes) & $14,13 \pm 14,83$ & $31,75 \pm 12,93$ & $0,050^{*}$ \\
\hline
\end{tabular}

${ }^{*} \mathrm{p}<0,05$ 
Valores de média \pm desvio dos dominios King's Health Questionnaire

\begin{tabular}{lccc}
\hline \multicolumn{1}{c}{ Domínio } & Pré & Pós & p \\
\hline Percepção geral de saúde & $40,63 \pm 18,60$ & $40,63 \pm 32,56$ & 1,000 \\
Impacto da incontinência & $66,64 \pm 35,64$ & $14,58 \pm 15,89$ & $\mathbf{0 , 0 1 8}^{*}$ \\
Limitaçóes das AVD's & $43,73 \pm 36,66$ & $10,40 \pm 23,44$ & $\mathbf{0 , 0 2 8}^{*}$ \\
Limitaçóes físicas & $45,81 \pm 40,58$ & $12,49 \pm 29,20$ & $\mathbf{0 , 0 2 8}^{*}$ \\
Limitaçóes sociais & $27,75 \pm 33,56$ & $6,25 \pm 17,68$ & 0,068 \\
Relaçốes Pessoais & $33,31 \pm 32,10$ & $4,16 \pm 11,77$ & $\mathbf{0 , 0 4 3}^{*}$ \\
Emoçôes & $59,71 \pm 45,60$ & $12,49 \pm 26,82$ & $\mathbf{0 , 0 2 8}^{*}$ \\
Sono e Disposição & $54,13 \pm 31,81$ & $16,65 \pm 19,91$ & $\mathbf{0 , 0 2 8}^{*}$ \\
Medida de Gravidade & $67,06 \pm 33,55$ & $25,80 \pm 30,93$ & $\mathbf{0 , 0 1 2}^{*}$ \\
\hline
\end{tabular}

${ }^{*} \mathrm{p}<0,05$

permitindo que o paciente mantenha a continência até o momento de chegar ao banheiro.

No presente estudo foi realizada contração ativa da MAP com a utilização do Biofeedback de pressão. Este recurso tem ação semelhante aos exercícios perineais utilizados no estudo supracitado, porém é mais adequado para os pacientes que não conseguem contrair adequadamente a musculatura do períneo.

Em contrapartida, em 1994, estudaram-se os efeitos do biofeedback eletromiográfico sobre a disfunção urinária de 20 voluntários com diagnóstico clínico de EM. Foram encontrados resultados significativos em relação ao pad test, escala análogo visual e episódios de perda urinária, porém sem relevância estatística ao se comparar com o grupo controle que não recebeu o tratamento ${ }^{17}$. Tal situação incentiva a realização de um grupo controle na presente pesquisa.

A melhora da força da MAP das voluntárias estudadas foi evidenciada através dos resultados obtidos na Avaliação Funcional do Assoalho Pélvico (AFA) e na Perineometria.

Em comparação com dados da literatura foi encontrado um estudo piloto realizado em 2006 com 35 mulheres portadoras de EM. Comparou-se os efeitos do treinamento ativo da MAP (grupo 1), do biofeedback eletromiográfico e do treino ativo da MAP (grupo 2) e da eletroestimulação, do biofeedback eletromiográfico e do treino ativo da MAP (grupo 3). O tratamento durou 24 semanas e foi constatado que a força muscular da MAP melhorou significantemente nos 3 grupos $(\mathrm{p}<0,05)$, porém sem diferença significante entre os grupos $(\mathrm{p}>0,146)^{18}$.

Posteriormente, em 2008, os mesmos autores realizaram estudo semelhante com 74 voluntários de ambos os sexos, com diagnóstico clínico de EM. Os voluntários foram divididos em dois grupos, sendo que o grupo 01 recebeu treinamento ativo da MAP, mais biofeedback eletromiográfico, mais eletroestimulação placebo; e o grupo 02 recebeu treinamento ativo da MAP, mais biofeedback eletromiográfico, mais eletroestimulação ativa. $\mathrm{O}$ tratamento durou 24 semanas e observou-se um aumento da força muscular nos dois grupos ( $\mathrm{p}=0,001)$, mas também sem diferença significativa entre eles $(\mathrm{p}=0,214)^{19}$.

Em relação aos resultados obtidos na presente pesquisa em relação à Perineometria, verifica-se a importância de se discutir a constituição da MAP. Ela é constituída por dois tipos de fibras: as do tipo I (lentas ou tônicas) e as do tipo II (rápidas ou fásicas). As do tipo I correspondem a $70 \%$ das fibras, possuem maior número de mitocôndrias e são mais resistentes à fadiga, importantes na manutenção da continência ao repouso. As do tipo II correspondem a $30 \%$ da MAP, caracterizam-se pela alta concentração de glicogênio e pela fadiga rápida, permitindo assim uma resposta rápida às diferenças súbitas de pressão $^{8,20,21}$.

Apesar do tratamento proposto enfatizar principalmente o treinamento de fibras tônicas ${ }^{21-23}$, pelo fato da paciente manter um tempo de contração prolongado (4 segundos), verificou-se uma melhora da força tanto das fibras tônicas (melhora do tempo da contração prolon- 
gada), quanto das fásicas (melhora da contração rápida máxima e da resistência). Tal resultado sugere que este tempo de contração também auxilie o fortalecimento de fibras fásicas, além das fibras tônicas.

Em comparação a dados da literatura, encontrase um artigo que analisa os valores obtidos na perineometria pré e pós intervenção com Biofeedback de pressão em mulheres com mais de 30 anos que apresentavam IU de esforço ou mista, porém sem bexiga neurogênica. Tais autores também encontram um aumento significativo da intensidade de contração muscular sustentada $(p<0,05)$, porém realizaram exercícios para fibras lentas ( 4 segundos de contração/ 4 segundos de repouso) e para fibras rápidas ( 2 segundos de contração/ 4 segundos de repouso ${ }^{23}$.

Outros autores também analisaram valores de perineometria pré e pós intervenção com biofeedback eletromiográfico em voluntárias com IU de esforço (também sem bexiga neurogênica). Aksac et al comparou os efeitos dos exercícios contra resistência digital e biofeedback eletromiográfico em 50 mulheres, tendo resultados estatis- ticamente favoráveis ao grupo submetido ao biofeedback, verificando-se valor de $\mathrm{p}<0,001$ na perineometria ${ }^{24}$. Rett encontrou um valor de $\mathrm{p}<0,0001$ na perineometria após o tratamento com cinesioterapia associado ao biofeedback eletromiográfico em 26 mulheres ${ }^{25}$.

Em análise à característica dos estudos supracitados, sugere-se a realização de novas pesquisas que verifiquem resultados da perineometria tanto em pacientes com desordens neurológicas, quanto em tratamentos com o Biofeedback de pressão para que haja maiores parâmetros de comparação com o presente estudo.

Quando se comparou a qualidade de vida através do King's Health Questionnaire antes e depois da intervenção, verificou-se que somente os domínios "Percepção Geral de Saúde" e "Limitaçôes Sociais" não possuíram diferenças significativas.

Podemos explicar a situação acima ao analisar as diversas limitaçóes que um paciente com EM pode enfrentar: fadiga, alteraçóes do equilíbrio e da estabilidade corporal, limitações funcionais (por exemplo: alterações

Tabela 4

Frequências absolutas e percentuais (entre parênteses) da escala de sintomas que consta no King's Health Questionnaire

Quanto que os problemas afetam você?

\begin{tabular}{|c|c|c|c|c|c|c|}
\hline Variável & Momento & Náo aplicável & Nenhum pouco & Um pouco & Moderadamente & Muito \\
\hline \multirow{2}{*}{ Frequência } & Pré & 0 & 0 & $3(37,5 \%)$ & $3(37,5 \%)$ & $2(25 \%)$ \\
\hline & Pós & $1(12,5 \%)$ & $4(50 \%)$ & $3(37,5 \%)$ & 0 & 0 \\
\hline \multirow{2}{*}{ Noctúria } & Pré & $2(25 \%)$ & $1(12,5 \%)$ & $3(37,5 \%)$ & $2(25 \%)$ & 0 \\
\hline & Pós & $4(50 \%)$ & $3(37,5 \%)$ & $1(12,5 \%)$ & 0 & 0 \\
\hline \multirow{2}{*}{ Urgência } & Pré & 0 & 0 & $2(25 \%)$ & $1(12,5 \%)$ & $5(62,5 \%)$ \\
\hline & Pós & 0 & $5(62,5 \%)$ & $2(25 \%)$ & $1(12,5 \%)$ & 0 \\
\hline \multirow{2}{*}{ Urgeincontinência } & Pré & $1(12,5 \%)$ & $1(12,5 \%)$ & $3(37,5 \%)$ & 0 & $3(37,5 \%)$ \\
\hline & Pós & 0 & $5(62,5 \%)$ & $2(25 \%)$ & $1(12,5 \%)$ & 0 \\
\hline \multirow{2}{*}{ IUE } & Pré & $2(25 \%)$ & 0 & 0 & 0 & $6(75 \%)$ \\
\hline & Pós & $2(25 \%)$ & $2(25 \%)$ & $4(50 \%)$ & 0 & 0 \\
\hline \multirow{2}{*}{ Enurese } & Pré & $4(50 \%)$ & $2(25 \%)$ & 0 & 0 & $2(25 \%)$ \\
\hline & Pós & $4(50 \%)$ & $4(50 \%)$ & 0 & 0 & 0 \\
\hline \multirow{2}{*}{$\begin{array}{l}\text { Incontinência } \\
\text { Relação Sexual }\end{array}$} & Pré & $3(37,5 \%)$ & $1(12,5 \%)$ & $2(25 \%)$ & $1(12,5 \%)$ & $1(12,5 \%)$ \\
\hline & Pós & $5(62,5 \%)$ & $3(37,5 \%)$ & 0 & 0 & 0 \\
\hline \multirow{2}{*}{$\begin{array}{l}\text { Infecçôes Urinárias } \\
\text { frequentes }\end{array}$} & Pré & $2(25 \%)$ & $3(37,5 \%)$ & $1(12,5 \%)$ & $2(25 \%)$ & 0 \\
\hline & Pós & $4(50 \%)$ & $4(50 \%)$ & 0 & 0 & 0 \\
\hline \multirow{2}{*}{ Dor na Bexiga } & Pré & $1(12,5 \%)$ & $2(25 \%)$ & $3(37,5 \%)$ & $1(12,5 \%)$ & $1(12,5 \%)$ \\
\hline & Pós & $3(37,5 \%)$ & $5(62,5 \%)$ & 0 & 0 & 0 \\
\hline \multirow{2}{*}{$\begin{array}{l}\text { Dificuldade para } \\
\text { Urinar }\end{array}$} & Pré & $1(12,5 \%)$ & $4(50 \%)$ & $1(12,5 \%)$ & $2(25 \%)$ & 0 \\
\hline & Pós & $4(50 \%)$ & $3(37,5 \%)$ & $1(12,5 \%)$ & 0 & 0 \\
\hline
\end{tabular}


da marcha e da função dos membros superiores ou inferiores), transtornos psicopatológicos como depressão, entre outros ${ }^{26,27}$.

Em virtude de tantas limitaçóes, nem sempre os sintomas urinários são os que mais incomodam o paciente. Sendo assim, mesmo atenuando os sintomas de urgência, frequência e/ ou urge-incontinência, o paciente permanece com outras limitaçóes que mantêm baixa sua percepção geral de saúde e ainda provocam limitaçóes sociais.

Vale ressaltar que apesar de a fadiga ser um fator limitante ao paciente com EM, as voluntárias foram capazes de realizar todos os procedimentos, também porque toda a metodologia foi estruturada respeitando essa limitação da população estudada.

Entre os domínios que apresentaram relevância estatística pós intervenção está o "Impacto da Incontinência” $(p=0,018)$. Isto significa que a intensidade do quanto o problema de bexiga afeta a vida das voluntárias diminuiu consideravelmente.

Em relação ao domínio "Limitação das Atividades de Vida Diária”, o resultado também foi significativo $(\mathrm{p}=0,028)$. Isto demonstra que os sintomas de urgência, frequência e/ou urge-incontinência inicialmente afetavam a realização de afazeres diários como fazer compras, limpar a casa e trabalhar ou realizar afazeres extra domésticos. Após a intervençáo, com a atenuação dos sintomas, tais atividades tornaram-se menos afetadas. Isto se explica pelo fato de que inicialmente, havia necessidade de constantes idas ao banheiro e ocasionalmente perdas urinárias o que comprometia a realização das tarefas.

Outro domínio que apresentou relevância estatística foi "Limitaçóes Físicas" ( $\mathrm{p}=0,028)$. Da mesma maneira descrita acima, as voluntárias inicialmente apresentavam limitaçôes para realizar atividades físicas como caminhar, correr, fazer ginástica, etc. Isto porque algumas voluntárias, além dos problemas característicos de bexiga hiperativa, apresentavam também incontinência urinária aos esforços, ou então, necessitavam parar as atividades para ir constantemente ao banheiro.

O domínio "Relações Pessoais" apresentou valor de $\mathrm{p}=0,043$ comparando-se pré e pós intervenção. Neste tópico destaca-se a relação das voluntárias com seus parceiros e o medo de perder urina durante a relação sexual. Além disto, sabe-se que a paciente com EM pode apresentar alteraçóes em sua sexualidade quanto à sensibilidade e/ou lubrificação vaginal ${ }^{28}$. Neste sentido a conscientização da MAP vem como um fator estimulante à relação sexual. Tal situação incentiva novos estudos mais aprofundados sobre a sexualidade do paciente com EM.

Em relação ao domínio "Emoçôes", o valor de $p$ foi de 0,028 . Com isto constata-se que o problema de bexiga na paciente com EM faz com que ela se sinta deprimida, ansiosa, nervosa e se sentindo mal consigo mesma. A partir do momento em que ela apresenta atenuação dos sintomas, ela se sente mais segura e confiante para realizar suas atividades.

A análise do domínio "Sono e Disposição" apresentou $\mathrm{p}=0,028$. Isto significa que a diminuição dos sintomas como urgência, frequência e/ou urge-incontinência permite melhores condiçóes de sono a paciente pela diminuição da noctúria.

Em relação ao domínio "Medidas de Gravidade", o valor de $\mathrm{p}$ foi de 0,012 . Neste domínio as voluntárias foram questionadas em relação à utilização de forros, do cuidado com a quantidade de líquido ingerida, da troca de roupas íntimas, da possibilidade de cheirar urina e do constrangimento por causa do problema de bexiga. Constatou-se que após o tratamento as voluntárias diminuíram a quantidade de medidas preventivas, o que demonstra a eficácia do procedimento realizado.

A análise das frequências absolutas e dos percentuais dos problemas relacionados à incontinência urinária que não constavam nos domínios do King's Health Questionnaire também demonstraram melhora dos sintomas. Houve diminuição da queixa de frequência urinária, da noctúria, da urgência, da urge-incontinência, da incontinência urinária aos esforços, da enurese, da incontinência durante a relaçáo sexual, da dor na bexiga e da dificuldade para urinar.

Apesar da análise subjetiva, os percentuais listados aos problemas acima confirmam de maneira positiva os resultados obtidos nos nove domínios do questionário.

Em estudo realizado com 155 portadores de EM para avaliação dos sintomas urinários, evidenciou-se que dentre os voluntários, 29,8\% apresentaram incontinência urinária de esforço, 70,2\% urgência, 61,7\% urgeincontinência, 36,2\% dificuldade para esvaziar a bexiga e $10,6 \%$ enurese $^{29}$. 
Em virtude da quantidade de correlaçóes positivas no presente estudo sugerimos continuidade da pesquisa. A idéia de comparar o efeito do biofeedback de pressão com o biofeedback eletromiográfico seria muito interessante, afinal o biofeedback de pressão possui uma valor mais acessível e é de mais fácil aplicação do que o biofeedback eletromiográfico. Tal situação facilitaria o acesso dos pacientes com EM aos profissionais devido ao baixo custo.

Sugere-se também a maior divulgação da pesquisa aos homens, os quais não aderiram ao presente estudo talvez pelo local de aplicação da sonda de biofeedback.

\section{CONCLUSÃO}

Verificou-se que o Biofeedback de pressão promoveu bons resultados no tratamento de pacientes com EM. Sua aplicação permitiu uma maior conscientização e força da MAP e melhora da qualidade de vida das pacientes.

A exceção de relevância estatística apresentada nos domínios de "Percepção Geral de Saúde" e "Limitaçôes Sociais" se deu devido aos diversos problemas que um paciente com EM pode enfrentar, os quais eventualmente são maiores do que seus sintomas urinários.

\section{AGRADECIMENTOS}

À Diretora das Clínicas Integradas, Denise Monteiro, pela disponibilização da área das Clínicas Integradas da Fepar para realização da pesquisa; Ao Dr. Gerson Fischer pelas orientaçôes metodológicas e de revisão da pesquisa; Ao Dr. Marcos Seefeld, pelo auxílio na captação de voluntários para a pesquisa.

\section{REFERÊNCIAS}

1. Rowland LP. Merrit: Tratado de Neurologia. 10 ed. Rio de Janeiro: Guanabara Koogan, 2000, 788p.

2.Fingerman JS, Finkelstein LH. The overactive bladder in multiple sclerosis. The Journal of The American Osteopathic Association 2000;100:9-12.

3.Baracho E. Fisioterapia Aplicada à Obstetrícia: Aspectos de Ginecologia e Neonatologia. 3 ed. Rio de Janeiro: Medsi, 2002, 626p.

4.Abrams P, Cardoso L, Fall M, Griffiths D, Rosier P, Ulmesten U. The standardization of terminology of lower urinary tract function: report from the standardization sub-committee of the international continence society. Urology 2003;61:37-49.
http://dx.doi.org/10.1016/S0090-4295(02)02243-4

5.Brugerolle B Chauvière C, André J.-M. Rétroaction biologique musculaire. Applications Du biofeedback dans les troubles moteurs. Encycl. Méd. Chir (paris-France), Kinésithérapie-Rééducation fonvtionnelle 1994;26:1-5. 6.Grosse D, Sengler J. Reeducação Perineal. São Paulo: Manole, 2002, 154p. 7.Bruschin I, Kano H, Damiāo R. I Consenso Brasileiro - Incontinência Urinária, Uroneurologia e Disfunçôes Miccionais. São Paulo: BG Cultural, 1999, $119 \mathrm{p}$.

8.Nascimento SM. Avaliação Fisioterapêutica da Força Muscular do Assoalho Pélvico na Mulher com Incontinência Urinária de Esforço após cirurgia de Wertheim-Meigs: Revisão de Literatura. Rev. Bras. de Canc 2009;55:157-63. 9.Capelini MV, Riccetto CL, Mambros M, Tamanini JT, Herrman V, Muller $\mathrm{V}$. Pelvic floor exercises with biofeedback for stress urinary incontinence. Int. braz j urol 2006;32:462-9.

http://dx.doi.org/10.1590/S1677-55382006000400015

10.Kalsi V, Fowle, CJ. Therapy Insight: bladder dysfunction associated with multiple sclerosis. Nat Clin Pract Urol 2005;10:492-501.

http://dx.doi.org/10.1038/ncpuro0323

11. Bo K, Finckenhagen HB. Vaginal palpation of pelvic floor muscle strength: inter-test reproducibility and comparison between palpation and vaginal squeeze pressure. J Urol 2003;169:2428-9.

12.Hundley AF, Wu JM, Visco AG. A comparison of perineometer to brink scores for assessment of pelvic floor muscle strength. Int Urogynecol J Pelvic Floor Dysfunct. 2005;192:1583-91.

13.Chiarapa TR, Cacho DP, Alves AFD. Incontinência Urinária Feminina: Assistência Fisioterapêutica e Multidisciplinar. São Paulo: Livraria Médica Paulista Editora, 2007, 225p.

14.Fonseca ESM, Camargo ALM, Castro RA, Sartori MGF, Fonseca MCM, Lima GR, et al. Validação do questionário de qualidade de vida (King's Health Questionnaire) em mulheres brasileiras com incontinência urinária. Rev Bras Ginecol Obstet 2005;27:235-42.

http://dx.doi.org/10.1590/S0100-72032005000500002

15.Mahony DT, Laferte RO, Blais DJ. Integral storage and voiding reflexes, neurophysiologic concept of incontinence and micturition. Urol 1977;9:95-106. http://dx.doi.org/10.1016/0090-4295(77)90297-7

16.Shafik A, Shafik IA. Overactive bladder inhibition in response to pelvic floor muscle exercises. World J Urol 2003;20:374-7.

17.Klarskov P, Heely E, Nyholdt I, Rottensten K, Nordenbo A. Biofeedback treatment of bladder dysfunction in multiple sclerosis. A randomized trial. Scand J Urol Nephrol (Suppl) 1994;157:61-5.

18.Mcclurg D, Ashe RG, Marshall K, Aowe-Strong AS. Comparison os Pelvic Floor Muscle Training, Electromyography Biofeedback, and Neuromuscular Electrical Stimulation for Bladder Dysfunction in People With Multiple Sclerosis: A Randomized Pilot Study. Neuroulogy an Urodynamics 2006;25:337-48. http://dx.doi.org/10.1002/nau.20209

19.Mcclurg D, Ashe RG, Lowe-Strong AS. Neuromuscular Electrical Stimulation an the Treatment of Lower Urinary Tract Dysfunction in Multiple Sclerosis - A Double Blind, Placebo Controlled, Randomised Clinical Trial. Neuroulogy an Urodynamics 2008;27:231-7.

http://dx.doi.org/10.1002/nau.20486

20.Figueiredo JA. Fisiologia vésico-esfincteriana. In: Montellato, N; Baracat, L; Arap, S. Uroginecologia. São Paulo: Roca, 2000, p.14-23.

21.Moreno AL. Fisioterapia em Uroginecologia. 2 ed. São Paulo: Manole, 2009, 240p.

22.Palma PC. Urofisioterapia: Aplicações clínicas das técniscas fisioterapêuticas nas disfunçốes miccionais e do assoalho pélvico. Campinas, SP: Personal Link Comunicaçôes, 2009, 532p.

23.Sanches PR, Silva DP Jr, Müller AF, Schmidt AP, Ramos JG, Nohama P. 
Vaginal probe transducer: characterization and measurement of pelvic-floor strength. J Biomech 2009;42:2466-71.

http://dx.doi.org/10.1016/j.jbiomech.2009.07.021

24.Aksac B, Aki S, Karan A, Yalcin O, Isikoglu M, Eskiyurt N. Biofeedback and pelvic floor exercises for the rehabilitation of urinary stress incontinence. Gynecol Obstet Invest 2003;56:23-7.

\section{http://dx.doi.org/10.1159/000072327}

25.Rett MT. Incontinência urinária de esforço em mulheres no menacme: tratamento com exercícios do assoalho pélvico associados ao biofeedback eletromiográfico [dissertação de mestrado]. Campinas: Universidade Estadual de
Campinas; 2004, 74p.

26.Almeida SEM, Bensuaski K, Cacho EWA, Oberg TD. Eficiência do treino de equilíbrio na Esclerose Múltipla. Fisioter. Mov 2007;20:41-8.

27.Lopes KN, Nogueira LAC, Nóbrega FR, Alvarenga-Filho H, Alvarenga RMP. Limitaçáo funcional, fadiga e qualidade de vida na forma progressiva primária da Esclerose Múltipla. Rev Neurocienc 2010;18:13-7.

28.Foley FW, Werner MA. Sexualidade. In: Kalb, RC. Esclerose múltipla: perguntas e respostas. São Paulo: Atlas, 2000, p.253-78.

29. Norton C, Chelvanayagam S. Bowel problems and coping strategies in people with multiple sclerosis. British Journal of Nursing 2010;19:220-6. 